Gdańsk 2019, Nr. 41

https://doi.org/10.26881/sgg.2019.41.04

Irina A. Schipowa (Ирина А. Шипова)

Staatliche Pädagogische Universität zu Moskau (Москва)

\title{
Textmodalität in den Texten von F. Kafka und F. Dostojewski aus kontrastiver Sicht
}

In dem Beitrag wird analysiert, wie einige Kategorien der Modalität in Texten von Kafka und Dostojewski realisiert werden und welchen pragmatischen Effekt sie produzieren können. Aus kontrastiver Sicht wird gezeigt, wie die Modalisierung vom Diskursmodell Erzählen im Deutschen und im Russischen erfolgen kann, welche Zielsetzung den Gebrauch von sprachlichen Mitteln die Modalität auf lexikalischer und morphosyntaktischer Ebene bestimmt, welche Modaloperatoren die Referenz der Modalität markieren und welches Zusammenwirken zwischen der Darstellungsart und dem Modalkern des Textes besteht.

Schlüsselwörter: Textmodalität, narrativer Diskurs, Erzählperspektive, kontrafaktische Modalität, epistemische Lesart

Modality of the text in the texts of F. Kafka and F.M. Dostoevsky in contrasting comparison. - The article analyzes how some categories of modality are realized in the texts of Kafka and Dostoevsky and what pragmatic effect they achieve. It is shown how modalization of the narrative discourse in the German and Russian languages takes place and what determines the purpose of using language means of expressing modality on the lexical and morph syntactic level, which modal operators mark the reference of modality and what is the relationship between the compositional narrative form of the narration and the modal core of the text.

Keywords: text modality, narrative discourse, narrative perspective, counterfactual modality, epistemic modality

Modalität des Textes wurde zum Gegenstand der Forschung in der sowjetischen Linguistik in den 80er Jahren des 20. Jahrhunderts in den Werken von GALPERIN (1981) und MosKaL'SKAÂ (1981). Die zentrale Schlussfolgerung dieser Forscher war, dass die Modalität des Satzes mit der des Textes nicht identisch ist. Darüber hinaus wurde hervorgehoben, dass bei der Bestimmung der modalen Charakteristika eines Textes die Spezifik der Textsorte relevant ist (MoskaL'SKaÂ 1981: 117). Derselben Meinung sind auch viele andere Linguisten (Turaeva 1986, Solganik 1999, Romanova 2003 u.a.).

Laut Moskal'skaâ spiegelt die Modalisierung eines Satzes die Gesetzmäßigkeiten der Textmodalität nicht wider, jeder Mikrotext enthält einen Modalkern, der nicht unbedingt der Modalcharakteristik eines Satzes entspricht. DieTRICH (1992: 37) zählt zum Kernbereich der Modalität den Ausdruck der Irrealität, der Möglichkeit, der Notwendigkeit und der damit verbundenen eingeschränkten Gewissheit. 
In Anlehnung an Galperin schreibt Romanova, dass die Textmodalität in ihrem Wesen objektiv und deswegen nicht ausschließlich eine grammatische Kategorie ist, sondern sie besitzt einen funktional-semantischen Charakter. So wird sie in verschiedenen Textpassagen nicht gleichmäßig ausgedrückt und wird in Abhängigkeit von der Intention des Textproduzenten von verschiedenen Faktoren beeinflusst (vgl. Romanova 2003). Das gilt für den schöngeistigen Text mehr als für andere Textsorten.

Die modale Komponente ist ein bedeutender Teil der Semantik des schöngeistigen Texttyps, die aus einer Kette von Satzmodalitäten entsteht und ihre eigene Hierarchie aufweist. Die Modalität der primären Ebene bilden die grammatischen Mittel, zu den Modalitäten der sekundären Ebene gehören die Erscheinungen, die den Text emotiv, expressiv oder ironisch färben und implizit die Einstellung des Textproduzenten zum Textinhalt ausdrücken (OtKupščıKova 1987: 47).

Die Organisation von Textinformationen in allen Referenzbereichen (auch in dem der Modalität) zeigt Strukturen, die mit der Diskursaufgabe zusammenhängen. Der schöngeistige Text ist in seinen Formen sehr mannigfaltig, deshalb kann in dieser Abhandlung nur auf eine prototypische Erzählung in der geschriebenen Sprache, nämlich auf die Narrativstruktur, eingegangen werden.

Die Diskursaufgabe des literarischen Narrativs ist die Verbalisierung von komplexen fiktionalen Sachverhalten, die chronologisch angeordnet, räumlich kohärent dargestellt sind, konsequent in Einzelereignisse zerlegt werden können und kunstvoll angeboten werden (vgl. Dietrich 1992: 113).

So ist in diesem Kontext der Diskurs als ein textübergreifender Verweiszusammenhang von isotopisch gebundenen Aussagen und als ein Mechanismus der Strukturierung dessen, was und wie gesagt und gedacht wird, definiert (vgl. WARNKE 2008: 40).

Zweck des Erzählens ist es, eine Geschichte so zu präsentieren, dass der Rezipient ihren Ablauf in seiner Vorstellung nachvollziehen und die Bewertung durch den Sprecher teilen kann (vgl. ZifonUn 1997: 123). Gegenstand des Erzählens ist es, einen Handlungszusammenhang mit dem objektiven Verlauf der Geschichte herzustellen. Die Wiedergabe folgt einer mentalen Organisation des Ablaufs, die der Vermittlung der Sprecherperspektive dient. Faktizität und Vollständigkeit sind dabei sekundär (ebd. 129).

Über die Sprecherperspektive entscheidet die Redeinstanz, die im literarischen Text als Erzählperspektive fungiert und in der traditionellen Klassifizierung von STANZEL (1987: 16) Ich-Erzählung (der Erzähler ist gleichzeitig Protagonist auf der Handlungsebene), auktoriale Erzählsituation (der Erzähler steht über der erzählten Welt und kommentiert als Autorität die fiktionale Welt) und personale Erzählsituation (die fiktionale Welt wird durch die Augen einer oder mehrerer Figuren geschildert) beinhaltet.

Laut PADUČEva (2010: 258) gebe es keinen literarischen Text, wo es keine Spuren seines Schöpfers gegeben hätte. Die Einstellung des Textproduzenten zum präsentierten Sachverhalt empfindet der Textrezipient in erster Linie durch die Modalität des Textes. Der qualitative Grad der modalen Ausdrücke in einem narrativen Text hängt aber mit dem Status des Erzählers (seiner expliziten oder impliziten Anwesenheit) zusammen. Die Erzählperspektive beeinflusst die Wahrnehmung des Rezipienten auf die Weise, dass die Reichweite des Dargestellten unterschiedlich spezifiziert sein kann: In Abhängigkeit von der Position des Betrachters bzw. 
der Redeinstanz ist ihm jeweils ein anderer Ausschnitt der externen Welt in der fiktionalen Wirklichkeit zugänglich. Die Erzählperspektive begrenzt die Menge des Wahrnehmbaren und der Autor des Textes gibt die Auswahl aus einer gegebenen Konfiguration vor, die er entweder verengt oder erweitert (vgl. STUTTERHEIM 1997: 24).

Je expressiver die Anwesenheit und die Beteiligung des Erzählers an der Handlungsebene markiert werden, desto ausgeprägter ist seine subjektive Stellungnahme zur erzählten Welt. Es hängt jeweils mit seinem Erzählstatus zusammen: Der auktoriale Erzähler zeigt die Ereignisse von allen Seiten, die erzählende Figur (auch der Ich-Erzähler) berichtet nur darüber, was sie selbst weiß, sieht bzw. gesehen und/oder erlebt hat. Dementsprechend wird das vermittelnde Subjekt mehr oder weniger wahrnehmbar.

Zwei weltbekannte Autoren: F. Dostojewski (1821-1881) und F. KAFKa (1883-1924) werden oft von den Literaturwissenschaftlern wegen der Themenähnlichkeit miteinander verglichen, aber es finden sich auch in der sprachlichen Gestaltung ihrer Texte bestimmte Gemeinsamkeiten, obwohl sie zu verschiedenen Epochen gehören und in verschiedenen Ländern gelebt und geschaffen haben.

In seinen Werken enthüllt „der bedeutendste Romancier seiner Zeit Dostojewski eine Unfähigkeit seiner Helden sich im Leben zurechtzufinden“, wobei er „die gesellschaftliche Misere dekuvriert und das verborgene Chaotische und Morbide aufspürt" (GlaSER 1997: 365, 366). Das künstlerische Bemühen, dem Erlebten und seiner seelischen Resonanz einen gemäßen gestalterischen Ausdruck im Text zu verschaffen, findet in sprachlicher Form seinen Niederschlag. Das als real Dargestellte mag durch bestimmte Techniken der Darstellung „objektiv unübersichtlich sein“ (ebd.: 363), und die Analyse zeigt, dass zu diesem Effekt im hohen Maße die Sprachmittel beitragen, die zum Ausdruck verschiedener modaler Entitäten eingesetzt werden.

In Kafkas Texten befindet sich der Mensch in einer Situation,

„die der in der Mitte eines langen Tunnels gleicht ohne Licht von vorn und von hinten, voller Verzweiflung und Angst [...]. So tastet der Mensch nach einem Weg und weiß über die Brüchigkeit der zeitgenössischen Welt, die keine Erklärung, keine Einsicht, geschweige denn eine Ordnung oder Idee vermittelt" (ebd.: 562, 563).

Diese „tönende Dunkelheit“ wird in Kafkas Werk dadurch besonders spürbar, dass seine fiktionale Realität wegen der hohen Frequenz modaler oder modal markierter Sprachmittel in die Sphäre des kaum Erklärbaren überführt wird.

Die Themen der beiden Schriftsteller, die sich in vielerlei Hinsicht kreuzen, finden ihren Ausdruck in Form von verschiedenen Erzählperspektiven. Dostojewski lässt in vielen seiner Romane den Ich-Erzähler sprechen, Kafka macht das auch, aber nicht oft. Meistens ist es formal gesehen die auktoriale Erzählsituation, in welcher der Erzähler über der fiktionalen Welt steht und sie als Autorität kommentiert. Aber in vielen Texten beider Autoren berichtet diese Redeinstanz darüber, was eine Romanfigur wahrnimmt, so dass der Text die Form der erlebten Rede annimmt, die eine Reflexionsdarstellung der Figuren ist, wenn sich die Perspektive des Autors und die der Figur decken, so dass eine gemischte Autor-Person-Perspektive entsteht (RIESEL 1975: 285). Dann fungiert eine Romanfigur zusammen mit dem Erzähler als vermittelndes Subjekt, was die Dominanz der epistemischen Lesart in vielen Textpassagen erklärt. 
In den narrativen Texten von F. Kafka und F.M. Dostojewski sind praktisch alle Arten der Modalität zu finden, denn der Ausdruck der Irrealität, der Möglichkeit, der Notwendigkeit oder der damit verbundenen eingeschränkten Gewissheit bildet die modalen Entitäten der fiktionalen Wirklichkeit von allen Seiten ab. Die Epistematizität als vorherrschende Art der Modalität nimmt ihre besondere Funktion wahr und hat wichtige pragmatische Aufgaben zu erfüllen.

Die Prosa von F. Kafka zeigt grundsätzlich gut erkennbare Tendenzen in der Darstellung der fiktionalen Wirklichkeit: Einerseits sieht der Textrezipient das normale, logisch geordnete Dasein der in Kafkas Werken auftretenden Figuren, andererseits passiert darin etwas, was dieses Dasein kardinal verändert und in den Bereich des Unerklärbaren überführt. Silman (1969: 254) nennt diese Art der Textproduktion „Traumtechnik“ und betont, dass nicht das Inhaltliche, sondern Methode und Stil des Erzählens dominant sind. Als eines der Mittel der Traumtechnik dienen konjunktivische Signale, die Modalität der Nichtfaktizität codieren.

Irreale Textsegmente, die durch verschiedene Formen des Konjunktivs markiert werden, gliedern den Text. Damit wird die auktorial vermittelte Realhandlung der Geschichte durch die Gedanken der Figur abgewechselt, so dass der Weltbezug außerhalb der eigentlichen Zeitachse des Narrativs situiert wird und der Sachverhalt als nicht realisierbar zu interpretieren ist. Der narrative Diskurs wird durch den Diskurs der Reflexion ersetzt und es entsteht die Doppelperspektive der auktorialen und der personalen Erzählsituation, wie in (1):

(1a) Flüchtig erinnerte sich K. an sein Heimatstädtchen; es stand diesem angeblichen Schlosse kaum nach. (1b) Wäre es $K$. nur auf die Besichtigung angekommen, dann wäre es schade um die lange Wanderschaft gewesen und er hätte vernünftiger gehandelt, wieder einmal die alte Heimat zu besuchen, wo er schon so lange nicht gewesen war. (Kafka: Das Schloss; Kursivdruck: I.S.)

Die konditionale Modalität in wenn-dann-Sentenz ( $1 \mathrm{~b})$ codiert irreale Bedingung und Folge. Beide bilden eine Einheit mit (1a) in der Textpassage, wo Autorenrede in erlebte Rede übergeht und die Erzählperspektive sich spaltet. Damit wird die Narration unterbrochen und die erzählte Welt bildet eine zusätzliche mentale Ebene ab, die eine Information über die Reflexion des Protagonisten beinhaltet.

Die gleiche oder ähnliche Funktion erfüllen die Modalitätsmarker der Vermutung, die die Propositionen in die Formate der eingeschränkten Gewissheit überführen und die Bestimmung der Redeinstanz erschweren. Ein Beleg dafür ist der Anfang des Romans Der Prozess (2), wo die Epistemizität den Kontrast zwischen vernünftiger Alltagsexistenz und neuer Lebensphase des Protagonisten markiert und die Weiterentwicklung der Ereignisse unbegreiflich macht.

(2) Jemand musste Josef K. verleumdet haben, denn ohne dass er etwas Böses getan hätte, wurde er eines Morgens verhaftet. Die Köchin der Frau Grubach, seiner Zimmervermieterin, die ihm jeden Tag gegen acht Uhr früh das Frühstück brachte, kam diesmal nicht. Das war noch niemals geschehen.

Im Falle, wenn die Erzählperspektive eindeutig auktorial geblieben wäre, sollte es wie in (2a) sein: 
(2a) Jemand hat Josef K. verleumdet, denn ohne dass er etwas Böses getan hat, wurde er eines Morgens verhaftet.

Der Vergleich von fast identischen Textpassagen (2) und (2a) zeigt, dass die zweite Variante die Erzählperspektive nicht spaltet und der darin abgebildete Sachverhalt keinen Zweifel zulässt.

Obwohl in (2) bei müssen der Grad der Wirklichkeit in der epistemischen Lesart sein Maximum erreicht, wird durch die Struktur Modalverb + Infinitiv II die Ungewissheit des Sachverhalts ausgedrückt, die durch den Gebrauch des Präteritum Konjunktiv des Verbs haben, des Indefinitpronomens jemand und den Kontrast in der Semantik der Adverbien diesmal und niemals implizit verstärkt wird. Auf diese Weise wird der auktoriale Erzähler nicht über die erzählte Welt gestellt, sondern es lässt sich die Stimme des Protagonisten hören, und dem Textrezipienten wird damit nur ein begrenzter Ausschnitt der fiktionalen Wirklichkeit zugänglich, was zur Bildung verschiedener Hypothesen inspiriert, den Eindruck des Geheimnisvollen schafft und Spannung erzeugt.

Zur Verstärkung der modalen Semantik von Wahrscheinlichkeit oder der Annahme im Text trägt die Kategorie der Unpersönlichkeit bei, die keine konkrete Person bezeichnet und in Texten von Kafka immer wieder in Form verschiedener Indefinitpronomen, unpersönlicher Sätze oder man-Konstruktion seine Aktualisierung findet und die bei der Textwahrnehmung zu allerlei Vermutungen provoziert. Dazu Beispiel (3):

(3) Im Nebenzimmer, in das K. langsamer eintrat, als er wollte, sah es auf den ersten Blick fast genau so aus wie am Abend vorher. Es war das Wohnzimmer der Frau Grubach, vielleicht war in diesem mit Möbeln, Decken, Porzellan und Photographien überfüllten Zimmer heute ein wenig mehr Raum als sonst, man erkannte das nicht gleich, um so weniger, als die Hauptveränderung in der Anwesenheit eines Mannes bestand, der beim offenen Fenster mit einem Buch saß, von dem er jetzt aufblickte.

Das Adverb fast, das die Charakteristik eines Sachverhalts nicht deutlich einstuft und subjektiv färbt, das in (3) erscheint und das Kafka im Allgemeinen oft gebraucht, codiert eine kaum wahrnehmbare Grenze zwischen dem, wie es ist und wie es sein sollte oder könnte. Seine Bedeutung erfüllt in gewissen Kontexten eine ähnliche Funktion, wie die Modalwörter und Modaladverbien, die dem Satz, in dem sie vorkommen, die epistemische Lesart verleihen. Belege dafür sind (3) und (4):

(4) [... ] Franz, [...] führte die Kaffeetasse, die er in der Hand hielt, nicht zum Mund, sondern sah K. mit einem langen, wahrscheinlich bedeutungsvollen, aber unverständlichen Blick an.

Zu bemerken sei auch, dass der von Kafka mit Vorliebe verwendete semantische Kontrast von Elementen mit gleicher syntaktischer Funktion (bedeutungsvoll, aber unverständlich) in (4) zur Verstärkung der modalen Bedeutung auf der sekundären Ebene beiträgt und dem Text den Eindruck einer beunruhigenden Fragwürdigkeit verleiht.

Eine besondere Rolle spielt in den Texten von Kafka die kontrafaktische Modalität, die „sich auf empirisch wahrnehmbare oder anders bekannte Sachverhalte bezieht, welche per se unmöglich sind“ (КотıN 2012: 143). Diese Modalitätsart wird durch irreale Komparativsätze codiert, deren Frequenz in Kafkas Texten außerordentlich hoch ist. Das gilt für den 
Roman Das Schloss, wo die Zahl solcher Sätze in einer Textpassage enorm hoch sein kann. Ein Beispiel dafür ist in (5) angeführt:

(5) Und als nun, ... der Kutscher quer über den Hof ging, [...] das große Tor zumachte, dann zurückkam, alles langsam und förmlich nur in Betrachtung seiner eigenen Spur im Schnee, dann sich im Stall einschloss und nun auch alles elektrische Licht verlöschte [...] und nur noch oben der Spalt in der Holzgalerie hell blieb und den irrenden Blick ein wenig festhielt, da schien es $K$., als habe man nun alle Verbindung mit ihm abgebrochen und als sei er nun freilich freier als jemals und könne hier auf dem ihm sonst verbotenen Ort warten, solange er wolle, und habe sich diese Freiheit erkämpft, wie kaum ein anderer es könnte, und niemand dürfe ihn anrühren oder vertreiben, ja kaum ansprechen; aber - diese Überzeugung war zumindest ebenso stark - als gäbe es gleichzeitig nichts Sinnloseres, nichts Verzweifelteres als diese Freiheit, dieses Warten, diese Unverletzlichkeit. (Kursivdruck: I.S.)

In (5) ist zu sehen, dass diese Strukturen in einer Äußerung mehrmals vorkommen und mit anderen Modalitätsmarkern eine Redundanz im Bereich der Epistemizität bzw. Kontrafaktizität schaffen, die den Traumeffekt produzieren. Die auktoriale Erzählperspektive wird auch hier durch die unmarkierte erlebte Rede abgewechselt, die Reichweite der Darstellung wird auf die Gedanken des Protagonisten konzentriert, deren Wahrnehmung den narrativen Diskurs in den Diskurs der Reflexion verwandelt und den Textrezipienten in eine andere mentale Dimension versetzt. Anhand des epistemischen Wertes wird eine mentale Entfremdung vom eigentlichen Inhalt hergestellt (vgl. LeIss 2009: 18). Die beiden modalen Entitäten dienen dem gleichen Ziel, die innere Spannung im Text zu schaffen und die Intrige des Inhalts zu verschärfen.

In Hypotaxen mit irrealen Komparativsätzen enthält oft der Matrixsatz das epistemische Prädikat scheinen, beides zusammen produziert eine Konvergenz der genannten modalen Entitäten. Die Form dieser Vergleichsätze geht von der Prämisse aus, dass die Proposition der Äußerung immer in Frage gestellt wird und die Akzeptabilität der Wahrnehmung durch die Vermutungen fraglich wird. Die epistemische Lesart wird deshalb dominierend.

Die Texte von Dostojewski, die einer anderen Epoche zugehören und in einer anderen Sprache verfasst wurden, zeigen hinsichtlich der Modalität viele Ähnlichkeiten mit denen von Kafka.

(6) Esli by kto teper vzglânul na nego iz prežde znavših ego polgoda nazad v Peterburge, v ego pervyj priezd, to, požaluj by, i zaklûčil, čto on naružnost û peremenilsâ gorazdo k lučšemu. No vrâd li èto bylo tak. ..., tak čto pri vnimatel'nom vzglâde na knâzâ sliškom bol 'šoj ohotnik posmeât'sâ, možet byt', i našel by, čemu ulybnut 'sâ. No malo li otčego byvaet smešno? (Der Idiot, 2. Teil, Kap. 2).

[Wenn einer von den Leuten, die ihn vor einem halben Jahre bei seinem ersten Aufenthalt in Petersburg kennengelernt hatten, ihn jetzt gesehen hätte, so würde er vielleicht gefunden haben, dass sein Äußeres sich sehr vorteilhaft verändert habe. Und doch war das kaum der Fall. [...], so dass ein besonders Lachlustiger bei einem aufmerksamen Blick auf den Fürsten vielleicht Anlass gefunden hätte zu lächeln. Aber was kommt den Leuten nicht alles lächerlich vor?]

Die Textpassage (6) aus dem Roman Der Idiot zeigt, dass man die gleichen Gesetzmäßigkeiten wie in (1) vorfindet, wo die Wirklichkeit mit der Irrealität konfrontiert wird. Die Autorenrede, 
die durch die Position des Betrachters einen bestimmten Ausschnitt der externen Welt in der fiktionalen Wirklichkeit präsentiert, unterbricht das Narrativ und spiegelt ein hypothetisches Bild wider, dass die Einstellung des auktorialen Erzählers zum Sachverhalt durch die Abbildung einer imaginären Situation im Konjunktiv etwas ironisch färbt. Auch hier codiert die konditionale Modalität der wenn-dann-Sentenz irreale Bedingung und Folge, die auf der Zeitachse des Narrativs bleibt. Die modalen Operatoren vryad li, požaluj und možet byt (,kaum, vielleicht ${ }^{\prime}$ ) verleihen dem ganzen Textabschnitt eine epistemische Lesart, womit die Faktizität des Sachverhalts in Frage gestellt wird. Die abschließende rhetorische Frage ist ein Marker von der Modalität der sekundären Ebene mit ironischer Bewertung des Erzählten.

Laut IvančEnkova (1973:354) ist im Text von Dostojewski der Charakter des Narrativs davon abhängig, was das Beschreibungsobjekt ist. Die Räume, Figurenporträts, Reiseberichte sind neutral und objektivierend verfasst. Deklarative Wortstellung und der damit einhergehende Textrhythmus entsprechen dem ruhigen Gang der Handlung wie in (7):

(7) General'ša [...] byla roslaâ ženŝina, odnih let s svoim mužem, s temnymi, s bol'šoû prosed'û, no eŝe gustymi volosami, s neskol'ko gorbatym nosom, suhoŝavaâ, $s$ želtymi, vvalivšimisâ ŝekami i tonkimi, vpalymi gubami. Lob ee byl vysok, no uzok; serye, dovol'no bol'šie glaza imeli samoe neožidannoe inogda vyraženie. (Der Idiot, 1. Teil, Kap. 5)

[Die Generalin [...] war eine hochgewachsene Frau, von gleichem Alter wie ihr Mann, mit dunklem, größtenteils schon ergrautem, aber noch dichtem Haar, etwas gekrümmter Nase, mager, mit gelben, eingefallenen Wangen und schmalen, welken Lippen. Ihre Stirn war hoch, aber nicht breit; die grauen, ziemlich großen Augen hatten mitunter einen recht ungewöhnlichen Ausdruck.]

Die Seelenbewegungen und Gemütszustände der Helden werden mit betonter Expressivität abgebildet, die durch entsprechendes emotiv geladenes Vokabular und eine Syntax der rhythmisch variabel gebauten Sätze ausgedrückt werden: Die Intonation der Textabschnitte wird teils dynamisch, teils statisch und ohne feste Gesetzmäßigkeiten. Die narrative Textstruktur wird oft durch rhetorische Fragen bzw. Exklamative unterbrochen (IvanČEnKOva 1973: 354). Obwohl diese Besonderheiten für die Narration im Allgemeinen nicht charakteristisch sind, sind sie zu der typischen Art der Textproduktion von Dostojewski geworden, die man in seinen Texten immer wieder vorfindet.

(8) No v poslednem slučae on prosto ne veril sebe i uprâmo, rabski, iskal vozraženij po storonam i oŝup'û, kak budto kto ego prinuždal i tânul k tomu. Poslednij že den', tak nečaânno nastupivšij i vsë razom porešivšij, podejstvoval na nego počti sovsem mehaničeski: kak budto ego kto-to vzâl za ruku i potânul za soboj, neotrazimo, slepo, s neestestvennoû siloj, bez vozraženij. Točno on popal kločkom odeždy v koleso mašiny, i ego načalo v nee vtâgivat'. (Schuld und Sühne, 1. Teil, Kap. 6)

[Aber bei diesem Resultate traute er einfach sich selbst nicht und tastete hartnäckig rechts und links nach neuen Einwendungen umher, als ob ihn jemand wie einen Sklaven dazu zwänge und anhielte. Der letzte Tag aber, der Tag, der so unerwarteterweise der letzte geworden war und alles mit einem Male zur Entscheidung gebracht hatte, hatte auf ihn fast völlig mechanisch gewirkt: wie wenn ihn jemand bei der Hand ergriffe und hinter sich herzöge, unwiderstehlich, blindlings, mit übernatürlicher Kraft, ohne Widerrede. Er war gleichsam mit einem Zipfel seiner Kleidung an einem Maschinenrade hängengeblieben, und dieses begann ihn in das Triebwerk hineinzuziehen.] 
Aus dem Beispiel (8) ist zu sehen, dass bei der Darstellung des inneren Zustands des Protagonisten die Kodierungsformen der Modalität die Expressivität des Textes intensivieren. Als Modaloperatoren treten in (8) die in der Russistik so genannten persuasiven Modalpartikeln kak budto, kak by (Toptygina 2011: 60), die im Deutschen den Konjunktionen der irrealen Komparativsätzen (als ob, als wenn, als) entsprechen und die modale Semantik der Kontrafaktizität wiedergeben. Die gleiche Semantik hat in (8) das Adverb točno in der Bedeutung etwa wie. Der Unterschied besteht darin, dass im Russischen diese Partikeln bzw. dieses Adverb nicht immer in einem Nebensatz aktualisiert werden und eine Proposition nur implizit enthalten.

Man findet in (8) auch das Adverb poctti (fast) in der Funktion der Charakteristik eines Sachverhalts als nicht deutlich und subjektiv eingeschätzt. Dieses Sprachmittel verwandelt in einem relativ kurzen Text das Narrativ in eine Vermutung der Redeinstanz über das Vorhandensein/Nicht-Vorhandensein eines Sachverhalts. Das Resultat davon ist der Eindruck der Unzulänglichkeit der rationalen Lebenswahrnehmung und Erklärung, die den Rezipienten in die innere Welt des Protagonisten versetzen und das Buch weiterlesen lässt.

Die Frequenz der Partikeln kak budto, kak by ist in den meisten Texten von Dostojewski enorm hoch. Die Textpassage (9) zeigt, wie diese Elemente dem Text den Charakter eines Traums verleihen:

(9) Stranno, lestnica byla kak budto znakomaâ! Von okno v pervom ètaže; grustno i tainstvenno prohodil skvoz' stekla lunnyj svet [...]. Šum ego sobstvennyh šagov ego pugal i trevožil. [...] V perednej bylo očen' temno i pusto, ni duši, kak budto vsë vynesli; [...] Vdrug poslyšalsâ mgnovennyj suhoj tresk, kak budto slomali lučinku, i vsë opât' zamerlo. Prosnuvšaâsâ muha vdrug s naleta udarilas' ob steklo i žalobno zažužžala. V samuû ètu minutu, i uglu, meždu malen'kim škapom i oknom, on razglâdel kak budto visâsiji na stene salop. [...] On podošel potihon'ku i dogadalsâ, čto za salopom kak budto kto-to prâčetsâ [...]. Vdrug emu pokazalos', čto dver' iz spal'ni čut'-čut' priotvorilas' i čto tam tože kak budto zasmeâlis' i šepčutsâ. (Schuld und Sühne, 3.Teil, Kap. 6).

[Merkwürdig: die Treppe kam ihm so bekannt vor! Da war das Fenster im Hochparterre; melancholisch und geheimnisvoll drang das Mondlicht durch die Scheiben; [...] Das Geräusch seiner eigenen Schritte erschreckte und ängstigte ihn. [...] Im Vorzimmer war es sehr dunkel und öde; keine Menschenseele war zu spüren; die Sachen schienen alle weggeschafft zu sein. [...] Plötzlich ertönte eine Sekunde lang ein trockenes Knacken, als ob jemand einen Holzspan zerbräche; dann wurde wieder alles still. Eine Fliege, die aufgewacht war, stieß beim Herumfliegen an die Fensterscheibe und summte kläglich. In demselben Augenblicke erblickte er in einer Ecke, zwischen einem kleinen Schränkchen und dem Fenster, einen Gegenstand, der wie ein an der Wand hängender Frauenmantel aussah [...] Er trat leise heran und erriet, dass sich jemand hinter dem Mantel versteckt hatte. [...] Auf einmal kam es ihm vor, als ob die nach dem Schlafzimmer führende Tür ein ganz klein wenig sich öffnete und auch dort gelacht und geflüstert würde.]

Außer den genannten Elementen hat (10) das epistemische Verb pokazalos', (auf einmal kam es ihm vor), andere unpersönliche Strukturen, nichtagensbezogene Konstruktionen und Indefinitpronomen [kto-to (jemand)], welche, wie gezeigt, auch in den Texten von Kafka vorhanden sind. Daraus kann man schließen, dass die Ähnlichkeit in den Texten der beiden Schriftsteller durch den Ausdruck der Modalität bedingt ist, obwohl Kafka sachlich, sogar nüchtern schreibt (vgl. Silman 1969: 261) und Dostojewski meistens expressiv mit deutlicher Emotivität seine Texte verfasst. 
Eine besondere Art, die Einstellung zum Inhalt des Textes zu codieren, ist in den Texten von Dostojewski zu finden: In seinen Beschreibungen erscheinen manchmal mehrere Substantive bzw. Adjektive in Verkleinerungsform. Das Diminutiv dient gewöhnlich zur Verniedlichung eines Objekts und bei seiner Anhäufung aktualisiert es das Verhältnis des Sprechers zu dem dargestellten Objekt. Solche Passagen können bei Dostojewski die Aussagen etwas ironisch färben, wie in (10):

(10) Akim Akimyč [...] povernulsâ pered krošečnym svoim zerkal'cem, kotoroe sobstvennoručno i davno uže okleil v svobodnuû minutku zolotym bordûrč̀kom. Tol'ko odin krûčoček u vorotnika kurtki okazalsâ kak budto ne na meste. Soobraziv èto, Akim Akimyč rešil perestavit' krûčok; perestavil, [...] i okazalos' uže sovsem horošo. Togda on složil vse i [...] uprâtal do zavtra v sundučok. (Aufzeichnungen aus einem toten Hause, Kap. 6)

[Akim Akimytsch grinste sogar vor Vergnügen, als er sich nicht ohne Eleganz vor seinem winzigen Spiegelchen drehte, den er in einer freien Stunde eigenhändig mit einer goldenen Bordüre beklebt hatte. Nur ein Häkchen am Kragen der Joppe schien nicht auf der richtigen Stelle zu sitzen. Als Akim Akimytsch es feststellte, entschloss er sich, das Häkchen zu versetzen; er versetzte es, [...] und stellte fest, dass nun alles tadellos saß. Darauf legte er die Sachen wieder zusammen und tat sie [...] in sein Köfferchen.]

Dieses Verfahren kann aber auch distanzierte Abwertung manifestieren, wie in (11):

(11) Ėtot Luka Kuz'mič byl tot samyj malen'kij, tonen'kij, s vostren'kim nosikom, moloden'kij arestantik našej kazarmy, iz hohlov, [...] Byl on v suŝnosti russkij, a tol'ko rodilsâ na ûge, kažetsâ, dvorovym čelovekom. V nem dejstvitel'no bylo čto-to vostroe, zanosčivoe: «mala ptička, da nogotok voster». (Aufzeichnungen aus einem toten Hause, Kap. 8)

[Dieser Luka Kusmitsch war der kleine, schmächtige, junge Arrestant aus unserer Kaserne, der Kleinrusse mit dem spitzen Näschen, [...] Eigentlich war er Großrusse, aber im Süden geboren, ich glaube als Leibeigener. Es war in ihm wirklich etwas Scharfes und Herausforderndes: Ein kleiner Vogel mit scharfen Krallen.]

Die Beispiele (10) und (11) zeigen die Modalität auf der sekundären Ebene, die emotive, expressive oder ironische Einstellung des Autors zu Objekten der dargestellten fiktionalen Wirklichkeit präsentieren kann.

Bei Kafka ist das Diminutiv ebenfalls zu finden, z.B. in der Erzählung Die Verwandlung:

(12) Nun ließ er sich gegen die Rückenlehne eines nahen Stuhles fallen, an deren Rändern er sich mit seinen Beinchen festhielt.

Aber es ist deutlich zu sehen, dass das in der Geschichte oft gebrauchte Diminutiv keine Modalität in den Text einbringt, sondern seine Nominalfunktion erfüllt.

Einige Besonderheiten der stilistischen Vorlieben der beiden Autoren (z.B. Diminutivgebrauch bei Dostojewski und kontrastive Semantik von gleichartigen Satzgliedern bei Kafka) schließen nicht die Ähnlichkeit in ihren Texten aus, was der Ausdruck der modalen Entitäten angeht.

Die Belege zeigen, dass es viel Gemeinsames in dem Stil der beiden Autoren gibt, was den Ausdruck der Modalität in ihren Texten angeht. Die ausschlaggebende Rolle gehört der Epistemizität, die den Äußerungen einen fraglichen, unheimlichen Nebensinn verleiht. Somit 
wird die Grenze zwischen dem Realen und Phantastischen undeutlich und das eine wird mit dem anderen untrennbar verflochten. Die mögliche Entwicklung des Sujets ist kaum zu prognostizieren, die Intrige bleibt erhalten und die Spannung lässt nicht nach. Gerade diese Eigenschaften der Werke der beiden Schriftsteller wecken das Interesse der Leser und machen ihr Schaffen so faszinierend.

\section{Quellen}

KafKa, Franz: Der Prozess.

KaFKa, Franz: Die Verwandlung.

KAFKA, Franz: Das Schloss, http://gutenberg.spiegel.de/autor/franz-kafka-309 (28.05.2018).

DostoevskiJ, F.M.: Idiot, https://ilibrary.ru/text/94/index.html (12.05.2018).

Dostoevskij, F.M.: Der Idiot, Übers. Hermann Röhl, https://gutenberg.spiegel.de/buch/deridiot-2098/1 (19.07.2019).

DostoevskiJ, F.M.: Prestuplenie i nakazanie, https://ilibrary.ru/text/69/index.html (12.05.2018).

Dostoevskij, F.M.: Schuld und Sühne, Übers. Hermann Röhl, https://gutenberg.spiegel.de/buch/ schuld-und-suhne-2100/1 (19.07.2019).

Dostoevskij, F.M.: Zapiski iz mertvogo doma, https://ilibrary.ru/text/61/index.html (12.05.2018).

Dostoevskij, F.M.: Aufzeichnungen aus einem toten Hause, Übers. Alexander Eliasberg, https:// gutenberg.spiegel.de/buch/aufzeichnungen-aus-einem-toten-hause-2092/1 (19.07.2019).

\section{Bibliographie}

Dietrich, Rainer (1992): Modalität im Deutschen: zur Theorie der relativen Modalität. Opladen: Westdeutscher Verlag.

Gal'perin, Il'â R. (1981): Tekst kak ob"ekt lingvističeskogo issledovaniâ. Moskva: Nauka.

Glaser, Hermann / Lehmann, Jakob / Lubos, Arno (1997): Wege der deutschen Literatur: eine geschichtliche Darstellung. Berlin: Ullstein.

IvANČENKOvA, Evgeniă A. (1973): Modal'nye tipy vyskazyvanij v hudožestvennyh tekstah Dostoevskogo i problema individual'nogo sintaksisa pisatelâ. In: Otazky slovanske syntaxe III. Brno, 353-356.

Kotin, Michail L. (2012): Modalitäten. In: Zeitschrift des Verbandes Polnischer Germanisten, $140-158$.

LEISS, Elisabeth (2009): Drei Spielarten der Epistemizität, drei Spielarten der Evidentialität und drei Spielarten des Wissens. In: ABraham, Werner / Leiss Elisabeth (Hg.): Modalität: Epistemik und Evidentialität bei Modalverb, Adverb; Modalpartikel und Modus. Tübingen: Stauffenburg Verlag, 3-24.

MoskaL'sKaÂ, Olga I. (1981): Grammatika teksta. Moskva: Vysšaâ škola.

Oт кupŝ́ıкоva, Maă I. (1987): Sintaksis svâznogo teksta: (strukturnaâ lingvističcskaâ model'). Dr.-Diss. Leningrad. 
PADUČEVA, Elena V. (2010): Semantika vremeni i vida v russkom yazyke. Semantika narrativa. Moskva: Jazyki slavânskoj kul'tury.

Riesel, Elise / Schendels, Elena (1975): Deutsche Stilistik. Moskau: Verlag Hochschule.

Romanova, Tatăna V. (2003): Modal'nost' kak tekstoobrazuûsaâ kategoriâ v sovremennoj memuarnoj literature. NGLU. Nižnij Novgorod, http://ucheb.lunn.ru/old/Publications/M_Romanova_Modalnost_2003.htm\#1-3 (26.05.2018).

Silman, Tamara I. (1969): Stilanalysen. Leningrad: Prosveŝenie.

SOLGANIK, Grigorij (1999): O tekstovoj modal'nosti kak semantičeskoj osnove teksta. In: Struktura i semantika hudožestvennogo teksta: Doklady VII Meždunarodnoj konferencii MGOPU. Moskva, 364-372.

StANZeL, Franz K. (1987): Typische Formen des Romans. Göttingen: Vandenhoeck \& Ruprecht.

StUtTerheim, Christiane (1997): Einige Prinzipien des Textaufbaus: Untersuchungen zur Produktion der mündlichen Texte. Tübingen: Niemeyer.

Toptygina, Elena N. (2011): Funkcii persuazivnyh edinic v tekste romana F.M. Dostoevskogo Prestuplenie i nakazanie. In: Vestnik Moskovskogo gosudarstvennogo oblastnogo universiteta 2011/1/, Russkaâ filologiâ, 59-63, www.evestnik.mgou.ru (28.05.2018).

Turaeva, Zinaida. (1994): Lingvistika teksta i kategoriâ modal'nosti. In: Voprosy âzykoznaniâ № 3, $105-115$.

Warnke, von Ingo H. (2008): Text und Diskurslinguistik. In: Janich, Nina (Hg.): Textlinguistik. 15 Einführungen. Tübingen: Narr, 35-54.

Zifonun, Gisela / Hoffmann, Ludger / Strecker, Bruno et al. (1997): Gramatik der deutschen Sprache. Berlin, New York: de Gruyter. 